\title{
CUATRO PUBLICACIONES DE LA COLECCIÓN STUDI GENTILIANI DEL CENTRO INTERNAZIONALE DI STUDI GENTILIANI DE SAN GINESIO (MACERATA) DURANTE EL TRIENIO 2018-2020
}

\author{
FOUR VOLUMES FROM THE STUDI GENTILIANI \\ COLLECTION, PUBLISHED BY THE CENTRO \\ INTERNAZIONALE DI STUDI GENTILIANI FROM SAN \\ GINESIO (MACERATA), DURING 2018-2020
}

\author{
Maria Valvidares Suárez \\ Universidad de Oviedo
}

\begin{abstract}
Recensión de / Review of: Stefano Colavecchia, Alberico Gentili e l'Europa. Storia ed eredità di un esule italiano nella prima età moderna, eum, Macerata, 2018, 225 págs.; AA.VV., Alberico e Scipione Gentili nell'Europa di ieri e di oggi. Reti di relazioni e cultura politica. Atti della Giornata Gentiliana in occasione del IV centenario della morte di Scipione Gentili (1563 - 1616); eum, Macerata, 2018, 215 págs.; Alberico Gentili, Libro di varie letture virgiliane al figlio Roberto (Lectionis Virgilianae Variae Liber. Ad Robertum filium, Hanau 1603). Introducción, traducción y anotaciones de Francesca Iurlaro; eum, Macerata, 2020, 369 págs.; AA.VV., Alberico Gentili e lo jus post bellum. Prospettive tra diritto e storia. Atti del convegno della XVIII Giornata Gentiliana. San Ginesio, 21-22 settembre 2018. A cura di Luigi Lacchè e Vincenzo Lavenia; eum, Macerata, 2020, 177 págs.
\end{abstract}

Palabras clave: Alberico Gentili, Scipione Gentili, ius belli, diritto diplomatico, diritto consolare, Centro Internazionale di Studi Gentiliani.

Key words: Alberico Gentili, Scipione Gentili, ius belli, diplomatic law, consular law, Centro Internazionale di Studi Gentiliani.

La presente reseña tiene por objeto dar cuenta de cuatro volúmenes publicados por el Centro Internazionale di Studi Gentiliani durante el trienio 20182020, bajo la presidencia del historiador Luigi Lacchè. Se trata, en particular, de cuatro nuevas obras que pasan a integrar la colección Studi Gentilliani, dirigida por los profesores Luca Scuccimarra, Paolo Palchetti y Vincenzo Lavinia, cuyo objeto es promover el conocimiento de la obra y biografia del humanista y jurista Alberico Gentili, considerado el fundador del moderno derecho internacional. La colección está cuidadosamente editada por eum: edizione università di Macerata.

1. Alberico Gentili e l'Europa. Storia ed eredità di un esule italiano nella prima età moderna. Stefano Colavecchia, eum, Macerata, 2018.

La primera de estas publicaciones, que se corresponde con el vol. 3 de la colección, recoge el trabajo del profesor de Historia Moderna de la Universidad de Molise, Stefano Colavecchia, bajo el título Alberico Gentili e l'Europa. Storia ed eredità di un esule italiano nella prima età moderna. En palabras de su autor, este 
estudio "se articula en torno a la historia del pensamiento de Alberico Gentili y de su herencia, influencia y fortuna en la Europa moderna". Según Colavecchia, la importante influencia del pensamiento del autor de De iure belli no fue suficientemente destacada por la historiografia italiana y europea, en parte porque el exilio de Alberico Gentili en Inglaterra, alejado de radicalismos, tuvo perfiles propios, lo que mantuvo su herencia intelectual fuera de las obras que glosaron el pensamiento de la mayoría de los exiliados italianos religionis causa del siglo XVI. El primer capítulo de este volumen profundiza en la trayectoria biográfica que lleva a Alberico Gentili desde San Ginesio, su localidad natal, hasta su nombramiento como profesor de derecho civil en Oxford a mediados de 1587, ya en el exilio.

La falta de atención a su obra a partir de la muerte de Gentili, "a pesar de la profundidad y amplitud de su reflexión" destacada por Colavecchia, y con la excepción de su "reaparición" en el marco del debate jurídico-político de la época de la Contrarreforma italiana en el siglo XVIII, no fue reparada hasta mediados del siglo XX. La referencia realizada en su obra $\mathrm{El}$ nomos de la tierra por el siempre controvertido jurista germano Carl Schmitt, sirvió para relanzar la figura de Gentili, si bien sería necesario esperar tres décadas para que viera la luz un estudio sistemático sobre el pensamiento de Alberico Gentili, firmado por el estudioso de la historia de la teoria política, Diego Panizza. Panizza ha sido, sin duda, una figura central en la promoción del conocimiento de la obra gentiliana, como ponen de manifiesto las palabras del prof. Luigi Lacchè, en el Preámbulo de otro de los volúmenes publicados en este trienio, quien se refiere a Panizza como el spiritus rector de dicho Centro.

Colavecchia destaca a lo largo del capitulo segundo de su obra la simbiosis entre el pensamiento de Alberico Gentili y el contexto histórico, cultural e institucional en el que se desarrolló, situado en los albores del nacimiento del nuevo estado moderno, gestado sobre el fracaso de la idea medieval de unidad de la cristiandad y la necesidad de pacificación de los conflictos religiosos. Gentili fue uno de los juristas, destaca Colavecchia, a los que se pidió "un esfuerzo de racionalización de la organización interna de los nuevos Estados", así como una nueva definición de la modalidad y las reglas de las relaciones internacionales que debía incluir un marco jurídico compartido "sobre la licitud del recurso a la guerra" entre los Estados. Según el historiador, Gentili rechaza sin matices la imposición de la fe por la fuerza, viendo en las guerras desencadenadas en nombre de la religión poco más que una tapadera para las ansias de dominio; si bien concibió al Estado como garante frente al extremismo religioso.

\section{Alberico e Scipione Gentili nell'Europa di ieri e di oggi. Reti di relazioni e cultura politica. Atti della Giornata Gentiliana in occasione del IV centenario della morte di Scipione Gentili (1563 - 1616); eum, Macerata, 2018.}

El volumen 4 de la colección Studi Gentiliani recoge, como su nombre indica, las Actas de las jornadas desarrolladas en San Ginesio los días 16 y 17 de septiembre de 2016, con ocasión del cuarto centenario de la muerte de Scipione Gentilli, hermano de Alberico. Se trata de seis intervenciones recogidas para su publicación en dos grandes bloques. 
El primer bloque recoge las intervenciones de la primera jornada, centrada particularmente en la trayectoria de Scipione Gentilli con especial atención a la red de relaciones intelectuales, politicas y religiosas en las que, por supuesto, también figura su hermano Alberico ("Sessione prima: Cultura, política e teologia al tempo dei Gentili").

La sesión se abre con el ensayo "Picenus hospes". Scipione Gentili interprete europeo della "Gerusalemme liberata", realizado por el profesor de Filosofia del Lenguaje de la Universidad de Roma Tre, Francesco Ferretti. En él, se da cuenta de la temprana notoriedad alcanzada por Scipione quien, con poco más de veinte años y ya en el exilio, realizó -en palabras del prof. Ferretti- el "experimento" de traducir al latín la obra Gerusalemme Liberata, de Torquato Tasso, de quien se convertiría en exégeta. Destaca Ferretti que la obra de Scipione, quien como su hermano contaba con formación jurídica, se inclinó hacia la poesía y en general la literatura, tal vez buscando así un espacio propio diferenciado del cultivado por Alberico. Por lo demás, Ferretti indica que, seguramente, Scipione contaba con beneficiarse del enorme prestigio del que gozaba la cultura italiana en la Inglaterra de la época.

El segundo ensayo del volumen, a cargo del profesor Cornel Zwierlein de la Universidad libre de Berlín, presta particular atención a lo que considera "particularidades" del método de Scipione Gentili, un acercamiento "filológicopoético" sobre el que el propio Gentili reflexionó en su obra Poeticae \& Jurisprudentiae adfinitas, y que se enmarca en el debate metodológico sobre los modos del conocimiento y la jerarquización de las disciplinas. Zwierlein, que enmarca a Scipione Gentili en la tercera generación dentro del humanismo jurídico, destaca en último término la importancia de las reflexiones de Gentili sobre el concepto de "conspiración", que en este sentido Zwierlein considera precursoras de la obra de su hermano Alberico.

Cierra esta “primera sesión” el trabajo del profesor Alberto Clerici, de la Università Niccolò Cusano de Roma, que indaga en el período que Scipione desarrolló en Holanda así como en el análisis de Scipione y Alberico Gentili sobre la revuelta de los Países Bajos contra España. Una época que resulta central, tal y como recuerda Clerici, en la construcción de un nuevo orden jurídico-político tras el derrumbe de la idea de la unidad medieval de la cristiandad. En su escrito, enmarcado en el contexto político y cultural de las "Provincias Unidas", Clerici profundiza en las relaciones que Scipione mantuvo con algunas de las personas más influyentes de la cultura holandesa del momento, integrantes del círculo del Conde de Leicester. Analiza, por último, el trabajo más relevante de Scipione durante el período holandés, destacado también en el primer ensayo de este volumen: las anotaciones a la obra de Torquato Tasso, Gerusalemme Liberata. Clerici enfatiza el hecho de que, a pesar de su posicionamiento personal a favor de la intervención de Inglaterra en auxilio de un pueblo oprimido desde el punto de vista político y religioso, Scipione no realiza en esta obra una defensa clara y explícita respecto de la licitud de la Revuelta. El autor enmarca esta ausencia en la indudable oposición de los hermanos Gentili a toda concepción de la soberanía popular -o contrato entre los súbditos y el príncipe- que pudiera significar una limitación de sus poderes, rechazo que, si bien parece dejar un cierto resquicio al ejercicio del derecho de resistencia, condena sin paliativos el tiranicidio. Para Clerici, la aparente contradicción entre la legitimación de la intervención inglesa y la negativa a reconocer la justa causa para los "rebeldes", se difumina si se tiene 
en cuenta, partiendo de las reflexiones de los Six livres de la République de Jean Bodin, que la legitimación se enmarca en una perspectiva internacional (un soberano que realiza una acción virtuosa) y el rechazo en un plano interno (los súbditos no pueden rebelarse legitimamente, pero sí pedir ayuda).

Los escritos de Claudia Storti, Eliana Augusti y Sir Michael Wood conforman la "segunda sesión": Le relazioni diplomatiche tra passato e presente, se centran en las relaciones entre el derecho consular y diplomático y el origen del ius inter gentes, cuestiones tratadas en la obra de los hermanos Gentili.

La profesora de Historia del Derecho Medieval y Moderno de la Università degli Studi di Milano - La Statale, Claudia Storti, se ocupa en su artículo L'officium legationis in età moderna, de reflexionar sobre diversas cuestiones que resultan conflictivas en el desarrollo del estatuto del embajador, definido como ciudadano en la encrucijada entre dos ordenamientos. Por un lado, se pregunta por las prerrogativas y privilegios que podía esperar del ordenamiento de acogida, en el que desarrollaba su officium sin dejar de ser extranjero. Por otro, atiende a la evolución de la figura del embajador desde su oficio público vinculado a la administración, hasta la consolidación de un perfil político-diplomático que consideraba al embajador como un "operador" de paz y amistad inter gentes.

Eliana Augusti, Profesora de Historia del Derecho Medieval y Moderno de la Università di Salento, aborda en su trabajo La giurisdizione consolare in Oriente: dal primato genovese alla sparizione. Spunti per una riflessione, la convivencia entre el derecho consular "común" y el "especial". El primero se identificaba con el derecho consular de los Estados europeos "cristianos y civiles". El segundo, también llamado derecho "capitular" o de las capitulaciones, era el que definía las relaciones con los paises al margen de la cultura occidental. En el marco de las relaciones capitulares, a una primera misión de cristianización de los "bárbaros" se sumó, posteriormente, el paradigma colonizador que protegió los intereses económicos de los Estados occidentales y "civilizados". Sobre esta asimetría se construyeron las jurisdicciones consulares occidentales en los Estados orientales, lo que no dejaba de implicar una suspensión de su soberanía, al no aplicárseles el aforismo "par in parem non habet jurisdictionem".

Cierra el volumen el escrito de Sir Michael Wood, miembro de la Comisión de Derecho Internacional y antiguo asesor legal del Foreign and Commonwealth Office, titulado: Diplomatic Law Today: Alberico Gentili Would No Have Felt our of Place. Destaca Sir Michael Wood el amplio abanico de intereses que Gentili desplegó en el campo del derecho internacional, desde el ius belli hasta el derecho del mar, pasando por el derecho de las relaciones diplomáticas, objeto central de estudio en este escrito -enmarcado, en este caso, en el derecho contemporáneo.

\section{Libro di varie letture virgiliane al figlio Roberto (Lectionis Virgilianae Variae Liber. Ad Robertum filium, Hanau 1603). Introducción,} traducción y anotaciones de Francesca Iurlaro; eum, Macerata, 2020.

Recoge el volumen 5 de la Colección que ocupa esta reseña la primera traducción de esta obra poco conocida de Alberico Gentili. Una obra que, en palabras de su traductora, ofrece nuevas perspectivas sobre la relación entre poesía y derecho y, más en general, sobre los fundamentos ideológicos y morales de la obra de Gentili. 
Este escrito, uno de los pocos que no tiene un perfil netamente jurídico, recoge los comentarios que Gentili realizaba para su hijo Roberto de la obra de Virgilio, las Bucólicas. Se ha considerado una muestra de su convicción en la importancia de los estudios humanísticos en la formación del jurista, al considerarlos un instrumento central para la identificación de los principios morales que debían guiar la interpretación de los textos legales. El nexo puede identificarse en la capacidad de la poesía para proporcionar ejemplos morales que el lector adaptaría para si mismo. Junto a ello, destaca Francesca Urlaro la preferencia que Gentili siente por Virgilio frente a Homero, al considerar que es su poesía la que encarna el ideal de justicia republicana, los valores de piedad y justicia que conforman la base normativa del ius gentium y que han de ser un referente para la educación de su hijo Roberto.

Identifica Francesca Urlaro tres ejes temáticos principales que Gentili desarrolla a lo largo de los veinte capítulos que conforman el Libro di varie letture Virgiliane: la relevancia de la gramática y la etimología en la educación; el uso de la poesía como fuente del derecho y la referencia a las figuras alegóricas de los pastores -a medio camino entre "bestialitas e humanitas"- como representación de las situaciones de estado de naturaleza y de civilización.

\section{Alberico Gentili e lo jus post bellum. Prospettive tra diritto e storia. Atti del convegno della XVIII Giornata Gentiliana. San Ginesio, 21-22 settembre 2018. A cura di Luigi Lacchè e Vincenzo Lavenia; eum, Macerata, 2020.}

La cuarta y última publicación destacada en esta reseña (el sexto volumen de la Colección Studi Gentiliani) recoge las actas del Congreso dedicado a Alberico Gentili e lo jus post bellum, que se desarrolló en San Ginesio en septiembre de 2018, en el marco de las "Jornadas Gentilianas" de celebración bienal. Jornadas de marcado carácter interdisciplinar con particular presencia de especialistas provenientes de la historia del derecho, la filosofía política y el derecho internacional y las relaciones internacionales. En este caso concreto -tal y como destaca en la presentación del volumen el profesor Luigi Lacchè, Presidente del Centro Internazionale di Studi Gentiliani-, el Congreso tenía por objeto de estudio la reconstrucción del debate histórico relativo al jus post bellum, es decir, a la relación entre el fin del conflicto bélico y la instauración de una paz justa, con una mirada atenta al cambio de paradigma de las guerras contemporáneas.

El volumen se estructura siguiendo las dos sesiones del Congreso. En la "primera sesión" se recogen los trabajos que centran su mirada en analizar las experiencias y reflexiones históricas del jus post bellum.

Abre la sesión el ensayo de Alain Wijffels, profesor en las Universidades de Leiden, Leuven y Louvain-la-Neuve, así como del Centre d'Histoire Judiciaire de Lille, titulado The jus post bellum, Cornerstone of Gentili's "De iure belli"?

El profesor Alain Wijffels desarrolla en su trabajo las razones por las cuales considera que el Libro III del De iure belli de Gentili, constituye la "piedra angular" de toda su obra. A su juicio, las cuestiones tratadas en este tercer libro, dedicado al jus post bellum, son aquellas en las que los juristas, en su papel de asesores politicos, podrian ejercer una mayor influencia. Del contenido del Libro III, relativo a las materias a abordar después de la guerra (venganza, satisfacción, 
compensación, tratados de paz...), Wijffels destaca el capítulo 12, que considera una "teoría condensada de la buena gobernanza aplicada a las situaciones postbélicas", en la que Gentili se ocupa de las relaciones, la tensión y las jerarquías entre los conceptos de utilidad, honor y justicia, que deben guiar las decisiones del buen gobierno con posterioridad a la guerra.

La segunda contribución lleva por título Quando la guerra è finita. Quale diritto per una pace giusta?, y su autor es el profesor de Historia del Derecho Medieval y Moderno de la Universidad de Salento, Luigi Nuzzo. En su escrito, revisa el papel de Gentili en la formación del moderno derecho internacional, construido sobre "la contraposición entre paz y guerra", de manera que la historia del derecho internacional "puede ser revisada asumiendo la guerra como paradigma narrativo". En el marco de la creación del concepto jurídico de jus post bellum, que permite ampliar el campo del derecho de la guerra tanto al período de transición que lleva del cese de hostilidades a la firma de un tratado de paz, cuanto las actividades de reconstrucción posteriores al acuerdo de paz, Nuzzo recuerda que para Gentili "el objetivo de la guerra era la realización de la paz". Frente a la interpretación que Carl Schmitt hace del pensamiento de Gentili, al señalarlo como representante del inicio de un derecho público europeo en el que el discurso de la guerra se libera de argumentaciones morales en beneficio del paradigma de soberanía estatal como justificación de la guerra justa, Nuzzo recuerda que, si bien para Gentili son los vencedores quienes determinan el contenido de la paz, también escribió que "ninguna paz puede ser justa y, por tanto, duradera, sin justicia". Incluso admitiendo el recurso a la guerra en el marco de las relaciones externas entre los Estados, Gentili aleja su regulación del modelo del derecho civil y la interpretación legal, para aproximarlo a los principios, costumbres e interpretaciones del paradigma del ius gentium, lo que impide romper la relación entre derecho y justicia.

Cierra esta primera sesión el trabajo de Alessandro Colombo, profesor de la Universidad La Statale de Milán, titulado Tra riscoperta della guerra giusta $e$ dissoluzione della guerra. La strada stretta dello jus post bellum, que abre el diálogo entre la obra de Gentili y los problemas contemporáneos. Colombo analiza las necesidades actuales del just post bellum desde el punto de vista de las conflictivas- relaciones entre derecho y política.

El escrito comienza manifestando cierta desconfianza sobre el propósito de un concepto como el de jus post bellum. Si en origen, las categorias y exigencias del jus ad bellum y del jus in bello ya venían condicionadas por la necesidad de no frustrar la posibilidad de la paz una vez terminada la guerra, no se ve clara la funcionalidad del ius post bellum. Le preocupa particularmente que, antes que señalar nuevos límites en el marco del derecho de la guerra, pueda servir para disculpar las vulneraciones del jus ad bellum "en nombre de la bondad de la paz" alcanzada. A partir de esta consideración, el trabajo de Colombo se pregunta tanto por el sentido práctico de la categoría de jus post bellum (¿cuánto tiene de jurídica? ¿tiene sentido la mezcla de contenidos que se acogen bajo esta etiqueta?), como por la cuestión histórica y conceptual que examina la inspiración y aspiraciones del propio concepto, en el que desaparecen las limitaciones vinculadas al respeto al status quo previo a la situación que legitimó la guerra. Ello se vincula con la tensión entre la perspectiva clásica del derecho inter-estatal y la perspectiva cosmopolita y humanitaria, y la correlativa tensión entre los principios de "orden" y de "justicia". 
La segunda sesión está dedicada a L'odierno diritto post-bellico: problemi e prospettive. Abre esta parte el trabajo de Giulio Bartolini, profesor de la Universidad de Roma-Tre, titulado Le riparazioni di guerra: da una prospettiva inter-statale ad una individuale. Bartolini estudia en primer lugar la cuestión de las reparaciones de guerra reconocidas en la obra de Alberico Gentili (recuperación de los gastos ocasionados por la guerra, reparación de daños, establecimiento de tributos...), pero también la praxis de los siglos XVII y XVIII, que muestran una reducción -incluso una renuncia- a la imposición de medidas económicas en la posguerra. Tendencia rota tras la Revolución Francesa y revalorizada durante el siglo XIX. En la posguerra de la I Guerra Mundial se reafirmó la obligación de reparar los gastos de guerra con base en el argumento de que la guerra les había sido "impuesta" por la agresión realizada por Alemania y sus aliados. El derecho a la reparación se reafirmó tras la II Guerra Mundial, si bien reconociendo expresamente el derecho de los vencidos a retener recursos suficientes para sostenerse de manera autónoma. En ambos casos es relevante destacar que el paradigma de la lógica estatal se vio complementado por la atención a los daños sufridos por las víctimas directas, la población civil.

Actualmente, la obligación de afrontar las reparaciones de guerra se vincula a la violación de las disposiciones de la Carta de las Naciones Unidas, previsión que -según Bartolini, que previamente había señalado un precedente en la IV Convención de La Haya de 1907- no ha gozado de gran aplicación práctica, destacando entre las causas el abandono de la firma de tratados de paz como herramienta entre los Estados en guerra, y la escasa incidencia de la "jurisdiccionalización de las controversias ligadas a los conflictos armados". Esta falta de realización práctica impide, a su juicio, que se hayan abordado con profundidad cuestiones relevantes al respecto, como el eventual carácter solidario de la exigencia de reparación, dado el carácter erga omnes de las obligaciones vulneradas; la concreción de la obligación de reparar cuando se vulnera la prohibición del uso de la fuerza (jus ad bellum) pero no las reglas del jus in bello o, en último término, el problema de la capacidad económica del Estado infractor.

Prosigue la segunda sesión el trabajo Mettere in discusione la stabilità delle situazioni territoriali illecite: l'obbligo di non riconoscimento nella prospettiva dello jus post bellum, firmado por Marco Pertile, profesor de Derecho Internacional en la Universidad de Trento. Su escrito comienza recordando el ambicioso objetivo del jus post bellum, que busca "definir un cuadro normativo coherente para la regulación de las situaciones internacionales vinculadas a la salida de los conflictos y al control del territorio en ausencia de soberanía". A partir de ahí, identifica una laguna en el debate contemporáneo, relativa a la problemática generada por el conflicto entre la ocupación efectiva de un territorio realizada con vulneración de las normas internacionales (la prohibición de conquista y el derecho de autodeterminación de los pueblos) y la obligación de no reconocimiento por parte de terceros Estados derivada, precisamente, de la ilicitud de la ocupación. Prohibición de reconocimiento que, a juicio del autor, forma parte de manera indubitada del jus post bellum.

Se pregunta Marco Pertile cuánto puede durar esta situación y cómo han de resolverse los problemas derivados de la prohibición de reconocimiento de quien ocupa de manera ilegal pero efectiva el territorio de un Estado. Destaca, a este respecto, una relativa praxis contemporánea en la que la tajante exigencia de retirada del territorio ocupado muta en una apelación a "negociar" una salida 
aceptable para ambas partes en conflicto. En todo caso, destaca Pertile in fine, la innegable prohibición de conquista presente en el derecho internacional contemporáneo supone una cesura con respecto del pensamiento y la época de Gentili, en la que se admitía la apropiación del territorio incluso como medida preventiva frente a un Estado que pudiera suponer una amenaza a la convivencia pacífica.

Cierra la segunda sesión, el sexto volumen y también esta extensa reseña, el trabajo de Samuel Wordsworth, especialista en Derecho Internacional Público y Arbitraje internacional y asesor gubernamental, Some Thoughts on jus post bellum before Domestic and International Courts. Sus reflexiones se articulan a partir de tres casos en los que el Gobierno británico fue parte implicada: Al-Saadoon and Mufdhi v. United Kingdom, Rahmatullah v. Ministry of Defence y, por último, Serdar Mohammed $v$. Ministry of Defence. A través de estos casos, Wordswoth analiza el eventual conflicto entre jurisdicciones de los diversos Estados participantes en una situación bélica; la justiciabilidad ante los Tribunales estatales de ciertos actos del gobierno británico y de sus agentes, entre los cuales no se encuentra el jus ad bellum o decisión de acudir a la guerra, residenciada en el Ejecutivo o en el Parlamento; y, por último, el derecho que debe ser aplicado por dichos Tribunales internos, dentro de un múltiple marco normativo que incluye el derecho internacional humanitario, el derecho del Estado en el que tiene lugar el acto objeto de análisis (en el caso analizado, una detención ilegal) y el Convenio para la protección de los Derechos Humanos y las Libertades públicas.

En resumen, cuatro extraordinarios e imprescindibles volúmenes para quien quiera profundizar no solo en el conocimiento de la biografia y la obra de Alberico Gentili, sino también en la proyección contemporánea de sus reflexiones, así como en el cambio de paradigma del derecho internacional respecto de su época.

Fecha de envío / Submission date: 18/04/2021

Fecha de aceptación / Acceptance date: 3/04/2021 